\title{
Ramadan Fast in Patients With Coronary Artery Disease
}

\author{
Mehdi Mousavi $^{1, *}$; SadafSadat Mirkarimi ${ }^{2}$; Gita Rahmani ${ }^{3}$; Ehsan Hosseinzadeh ${ }^{2}$; Navid \\ Salahi ${ }^{2}$ \\ ${ }^{1}$ Shahid Bahonar Hospital, Alborz University of Medical Sciences, Karaj, IR Iran \\ ${ }^{2}$ Khatam Al-Anbia Hospital, Islamic Azad University, Shahroud, IR Iran \\ ${ }^{3}$ Private Clinic, Karaj, IR Iran \\ *Corresponding Author: Mehdi Mousavi, Shahid Bahonar Hospital, Alborz University of Medical Sciences, Karaj, IR Iran. Tel:+98-2632527575, Fax:+98-2632546374, E-mail:mmoosavi@ \\ razi.tums.ac.ir
}

Received: August 25, 2012; Revised: January 17, 2014; Accepted: October 25, 2014

\begin{abstract}
Background: Fasting during the month of Ramadan is of vital significance amongst Muslims; however, little is known about the effects of this kind of fasting on patients with coronary artery disease (CAD).

Objectives: This nonrandomized prospective observational pilot study was designed to investigate the effects of Ramadan fast on the symptoms of CAD.

Patients and Methods: Patients with documented CAD were consecutively (nonrandomized) included in the study, and those with heart failure (ejection fraction $<50 \%$ ), renal failure, gout, and insulin-treated diabetes were excluded. Patients had the choice of fasting during Ramadan if they so wished and to break their fast as soon as symptoms such as dyspnea and chest pain occurred (fasting group) or not fasting (control group).

Results: A total of 148 patients completed the study. Mean (mean \pm SD) age of the patients was $61.5 \pm 11.7$ years and $50 \%$ were male. Finally, 66 patients (44.6\%) accomplished Ramadan fast with an average of $22.27 \pm 10.46$ days of fasting. Occurrence of chest pain was not significantly different between the fasting and non-fasting groups (4 out of 66 [6.1\%] vs. 8 out of 82 [9.8\%] respectively; $\mathrm{P}=0.42$ ). In addition, patients who fasted during Ramadan did not experience a higher frequency of a combined endpoint of chest pain and dyspnea ( 4 out of 66 cases in the fasting group [6.1\%] vs. 11 out of 82 in non-fasting group [13.4\%]; $\mathrm{P}=0.14$ ).

Conclusions: In the present study, the patients with CAD were able to observe Ramadan fast safely and their combined endpoint of chest pain and dyspnea was not significantly different from that of the non-fasting ones. We would suggest that patients with CAD and normal left ventricular function could fast during Ramadan.
\end{abstract}

Keywords: Fasting; Coronary Artery Disease; Ramadan; Chest Pain; Dyspnea

\section{Background}

Ramadan is the holiest month of the Islamic calendar, mandating healthy adult Muslims to eschew from sinful speech and behavior and observe abstinence from eating and drinking, including taking oral or intravenous medication, from dawn to dusk (1). During Ramadan, Muslims tend to eat two meals: a pre-fast meal before sunrise (Sahar or Suhoor) and a meal shortly after sunset (Iftaar). This obligatory change in lifestyle, in terms of obtaining the daily calorie intake via two meals in lieu of the customary four or five meals a day or alteration in medication schedule or reduction in daily sleep duration, might affect patients with cardiovascular diseases (CVD). Although more than a billion Muslims observe Ramadan fast the world over, there is still a great deal of controversy in the medical community on the effect of this important Islamic tenet on CVD (2-4) and there is a dearth of data on its impact on patients with coronary artery disease (CAD).

\section{Objectives}

The purpose of this prospective observational study was to determine whether fasting in Ramadan has any effect on the symptoms of patients with stable CAD.

\section{Patients and Methods}

thirty days prior to the commencement of Ramadan, outpatients with stable CAD, documented by angiography, exercise test, or single photon emission computed tomography (SPECT), were consecutively (nonrandomly) selected to participate in the study, if they provided written informed consent. Patients with systolic left ventricular dysfunction (ejection fraction $[\mathrm{EF}]<50 \%$ ), renal failure, gout, and diabetes mellitus (DM) requiring insulin or uncontrolled DM were excluded. Among 349 documented patients with stable CAD that were visited in the mentioned period, 148 patients signed the written informed consent and were included.

The study population were given the choice to fast

Copyright (C) 2014, Iranian Red Crescent Medical Journal. This is an open-access article distributed under the terms of the Creative Commons Attribution-NonCommercial 4.0 International License (http://creativecommons.org/licenses/by-nc/4.0/) which permits copy and redistribute the material just in noncommercial usages, provided the original work is properly cited. 
Mousavi Met al.

\begin{tabular}{|c|c|c|c|c|}
\hline Variable & Total, $\mathrm{n}=148$ & Ramadan Fasting Group, $n=66$ & Non-Fasting Group, $\mathrm{n}=\mathbf{8 2}$ & P Value \\
\hline Age, $y$ & $61.47 \pm 11.70$ & $59.26 \pm 10.55$ & $63.26 \pm 12.32$ & 0.038 \\
\hline Male Sex & $74(50 \%)$ & $35(53.0 \%)$ & $39(47.7 \%)$ & 0.508 \\
\hline Diabetes mellitus & $36(24.3 \%)$ & $12(18.2 \%)$ & $24(29.3 \%)$ & 0.118 \\
\hline Hypertension & $91(61.5 \%)$ & $37(56.1 \%)$ & $54(65.9 \%)$ & 0.224 \\
\hline Systolic Blood Pressure, mm Hg & $132 \pm 22$ & $132.1 \pm 23$ & $132 \pm 21$ & 0.978 \\
\hline Diastolic Blood Pressure, mm Hg & $75 \pm 10$ & $75.2 \pm 9.5$ & $75.4 \pm 10.1$ & 0.883 \\
\hline History of CABG & $32(21.6 \%)$ & $9(13.6 \%)$ & $23(28.1 \%)$ & 0.034 \\
\hline History of PCI & $31(20.95 \%)$ & $17(25.8 \%)$ & $14(17.07 \%)$ & 0.197 \\
\hline Diuretics Intake & $21(14.2 \%)$ & $8(12.1 \%)$ & $13(15.9 \%)$ & 0.542 \\
\hline Nitrates Intake & $107(73.3 \%)$ & $47(71.2 \%)$ & $60(73.2 \%)$ & 0.971 \\
\hline Beta-Blockers Intake & $122(83.6 \%)$ & $57(86.4 \%)$ & $65(79.3 \%)$ & 0.113 \\
\hline ACE Inhibitors Intake & $62(42.5 \%)$ & $25(37.9 \%)$ & $37(45.1 \%)$ & 0.462 \\
\hline Calcium Channel Blockers Intake & $55(37.7 \%)$ & $21(31.8 \%)$ & $34(41.5 \%)$ & 0.284 \\
\hline \multicolumn{5}{|l|}{ Treatment Category } \\
\hline MT & $85(57.4 \%)$ & $40(60.61 \%)$ & $45(54.88 \%)$ & 0.484 \\
\hline PCI-MT & $16(10.8 \%)$ & $8(12.12 \%)$ & $8(9.76 \%)$ & 0.645 \\
\hline PCI-No CP & $15(10.1 \%)$ & $9(13.64 \%)$ & $6(7.32 \%)$ & 0.205 \\
\hline CABG-MT & $15(10.1 \%)$ & $4(6.06 \%)$ & $11(13.41 \%)$ & 0.141 \\
\hline CABG -No CP & $17(11.5 \%)$ & $5(7.58 \%)$ & $12(14.63 \%)$ & 0.302 \\
\hline MT+PCI-MT + CABG-MT & $116(78.4 \%)$ & $52(78.8 \%)$ & $64(78.0 \%)$ & 0.914 \\
\hline
\end{tabular}

(fasting group) or not to fast (control group) during Ramadan. In the period of our study (August 12, 2010 through September 9, 2010) and in our location (Shahroud, Iran), Ramadan fell in the summer season: the temperature was $23^{\circ} \mathrm{C}$ to $35^{\circ} \mathrm{C}$ during the fasting time and fasting lasted for about 13.5 to 14 hours a day. The fasting patients were advised to break their fast as soon as they experienced symptoms such as chest pain, dyspnea, and severe palpitation and take their medications (eg, TNG). All the cardiovascular drugs were adjusted and continued twice daily (at Sahar and Iftaar), and the patients were visited up to thirty days after the termination of Ramadan and asked about days of fasting and possible symptoms during fasting. If the patient was admitted to hospital or there was a need for visit due to change in the symptoms during the study period, suitable treatment would be performed according to current guidelines.

The patients were assigned to five treatment categories:

1. Medical treatment: Those who were candidate for medical treatment because of unsuitable coronary anatomy for revascularization; those who were candidate for medical follow-up because of acceptable response to medical treatment; and those suitable for revascularization but unwilling to undergo this option.

2. Patients with a history of percutaneous coronary intervention (PCI) and typical chest pain who were candidate for medical treatment.

3. Patients with a history of PCI without typical chest pain after PCI.
4. Patients with a history of coronary artery bypass graft surgery (CABG) and typical chest pain who were candidate for medical treatment.

5. Patients with a history of CABG with no typical chest pain after $C A B G$.

Local Ethics Committee in Islamic Azad University, Faculty of medicine, Khatam Al-Anbia Hospital, approved the study (ethical approval code: 284; April of 2010).

\subsection{Statistical Analysis}

The data were analyzed using SPSS 16 (SPSS Inc., Chicago, IL). The data were expressed as mean \pm standard deviation (SD) and numbers (\%). The Student's t test was used to evaluate the significance of the differences between the mean values of two continuous variables, and the MannWhitney U test was employed if the distribution was not normal. Chi square analysis was performed to test for differences in the proportions of the categorical variables between two or more groups, and if necessary, the Fisher's Exact test (two-tailed) was utilized instead of Chi square. Logistic regression tests were performed to evaluate the significance of Ramadan fast as a predictor of chest pain or a combined endpoint of chest pain and dyspnea. Variables with a P value of $<0.1$ were included in the model, and $P$ value $<0.05$ was considered statistically significant.

\section{Results}

A total of 148 patients with stable CAD, with the mean age 
Table 2. A Comparison of Chest Pain or Combination of Chest Pain and Dyspnea in the Study Groups According to the Mode of Treatment ${ }^{\mathrm{a}}$

\begin{tabular}{lcccccc}
\hline Symptom & Total & Fasting Group & Non-Fasting Group & P value & OR & 95\% CI for OR \\
\hline Chest Pain (Total) & $12 / 148(8.1 \%)$ & $4 / 66(6.1 \%)$ & $8 / 82(9.8 \%)$ & 0.413 & 0.597 & $0.172-2.076$ \\
\hline Chest Pain or Dyspnea (Total) & $15 / 148(10.1 \%)$ & $4 / 66(6.1 \%)$ & $11 / 82(13.4 \%)$ & 0.141 & 0.126 & $0.126-1.374$ \\
\hline Chest Pain in MT + PCI-MT + CABG-MT & $12 / 116(10.3 \%)$ & $4 / 52(7.7 \%)$ & $8 / 64(12.5 \%)$ & 0.398 & 0.583 & $0.165-2.058$ \\
$\begin{array}{l}\text { Chest Pain or Dyspnea in MT + PCI-MT } \\
\text { + CABG-MT }\end{array}$ & $15 / 116(12.9 \%)$ & $4 / 52(7.7 \%)$ & $11 / 64(17.2 \%)$ & 0.130 & 0.402 & $0.120-1.345$ \\
\hline Chest Pain in MT & $7 / 85(8.2 \%)$ & $3 / 40(7.5 \%)$ & $4 / 45(8.9 \%)$ & $>0.999$ & 0.831 & $0.174-3.961$ \\
\hline Chest Pain or Dyspnea in MT & $8 / 85(9.4 \%)$ & $3 / 40(7.5 \%)$ & $5 / 45(11.1 \%)$ & 0.717 & 0.649 & $0.145-2.906$ \\
\hline Chest Pain in PCI-MT & $3 / 16(18.8 \%)$ & $1 / 8(12.5 \%)$ & $2 / 8(25 \%)$ & $>0.999$ & 0.429 & $0.031-5.985$ \\
\hline Chest Pain or Dyspnea in PCI-MT & $4 / 16(26 \%)$ & $1 / 8(12.5 \%)$ & $3 / 8(37.5 \%)$ & 569 & 0.238 & $0.019-3.011$ \\
\hline Chest Pain in PCI-No CP & $0 / 15(0)$ & $0 / 9$ & $0 / 6$ & $>0.999$ & - & - \\
\hline Chest Pain or Dyspnea in PCI-No CP & $0 / 15(0)$ & $0 / 9$ & $0 / 6$ & $>0.999$ & - & - \\
\hline Chest Pain in CABG-MT & $2 / 15(13.3 \%)$ & $0 / 4$ & $2 / 11(18.2 \%)$ & $>0.999$ & 0 & - \\
\hline Chest Pain or Dyspnea in CABG-MT & $3 / 15(20 \%)$ & $0 / 4$ & $3 / 11(27.3 \%)$ & 0.516 & 0 & - \\
\hline Chest Pain in CABG-No CP & $0 / 17(0)$ & $0 / 5$ & $0 / 12$ & $>0.999$ & - & - \\
\hline Chest Pain or Dyspnea in CABG-No CP & $0 / 17(0)$ & $0 / 5$ & $0 / 12$ & $>0.999$ & - \\
\hline
\end{tabular}

a Abbreviations: OR, odds ratio; CI, confidence interval; CABG-MT, patients with history of coronary artery bypass graft (CABG) who had history of chest pain, but were treated medically; CABG-no CP: patients with history of CABG who had no typical chest pain; MT, patients who were on medical treatment; PCI-MT, patients with history of percutaneous coronary intervention (PCI) who had history of chest pain, but were treated medically; PCI-no CP, patients who were on medical treatment; and PCI-MT, patients with history of PCI who had no typical chest pain.

of $61.5 \pm 11.7$ years, completed the study. Males accounted for $50 \%$ of the study population. Sixty-six patients (44.6\%) fasted during Ramadan for an average of 22.27 \pm 10.46 days. The basic characteristics of the fasting and non-fasting groups are summarized in Table 1.

During the study follow-up period, two patients in the non-fasting group and one in the fasting group were admitted to the hospital because of severe symptoms. Information on the occurrence of chest pain and a combination of chest pain and dyspnea in study groups is provided in Table 2 . Chest pain occurred in 12 patients (8.11\%) during Ramadan; the occurrence of chest pain was not significantly different between study groups with four patients (6.1\%) in fasting group and $8(9.8 \%)$ in nonfasting group $(\mathrm{P}=0.413$; odds ratio $[\mathrm{OR}]=0.597$; and $95 \%$ confidence interval [CI], 0.172-2.076).

The combined endpoint of chest pain and dyspnea occurred in four patients (6.1\%) in the fasting group and $11(13.4 \%)$ in the other group $(\mathrm{P}=0.141, \mathrm{OR}=0.416$, and 95\% CI, 0.126-1.374). Chest pain trended to occur more frequently amongst women with nine women (12.2\%) and three men $(4.1 \%)$ presenting with chest pain ( $\mathrm{P}=$ $0.071, \mathrm{OR}=3.277$, and 95\% CI, 0.850-12.631). With respect to sex, the combined endpoint of chest pain and dyspnea occurred in 11 women (14.9\%) and four men (5.4\%) $(\mathrm{P}=0.057, \mathrm{OR}=3.056$, and 95\% CI, 0.926-10.083). Our multivariate analysis confirmed that Ramadan fast was neither a predictor of chest pain nor a combination of chest pain and dyspnea.

\section{Discussion}

Almost half of our study population (44.6\%) managed to fast during Ramadan. There was no significant difference in the proportion of chest pain and a combined endpoint of chest pain and dyspnea between our participants (Table 2). These results were still the same even after adjusting our data for age and other possible confounding variables. Furthermore, our medically treated patients as well as those with prior PCI and CABG who had a history of chest pain and were medically treated did not experience a higher frequency of chest pain or a combined endpoint of dyspnea and chest pain. Chest pain or the combined endpoint of chest pain and dyspnea were not significantly different between the treatment categories (Table 2). In fact, none of the patients with a history of revascularization, including PCI and CABG, who had no history of post-revascularization typical chest pain reported chest pain during the month of Ramadan. In this subgroup of our study population, fasting was safe; consequently, patients with a history of revascularization but without heart failure as well as a history of post-revascularization chest pain should not be afraid to fasting; however, the relatively small sample size of our study renders it underpowered and further studies are needed to clarify the safety of Ramadan fast in this population.

The results of the present study chimed in with those of some other studies in the existing literature. In a systematic review of literature, Salim et al. stated that during Ramadan fasting, the incidence of acute cardiac illness 
was not different from non-fasting days and most of the patients with stable disorders of heart could fast during Ramadan without any clinical deterioration. In comparison to non-fasting days, there might be a difference in the timing of symptom onset and events might significantly increase during the period of "Iftaar" (5). Al Suwaidi et al. (3) studied 465 patients with different stable heart diseases including heart failure, atrial fibrillation, and valvular disease who observed Ramadan fast (288 patients [62\%] had angina) and reported that $91.2 \%$ of the patients were able to fast and only $6.7 \%$ felt worse during Ramadan fast. Chamsi-Pasha and Ahmed (6) recruited 86 outpatients with different kinds of heart disease (46 patients [53\%] with CAD) and reported that 74 patients (86\%) succeeded in fasting for the whole duration of Ramadan, while 9 (10.4\%) missed the fasting for up to seven days and $3(3.5 \%)$ could not fast at all. There were no significant changes in the New York Heart Association Class $(\mathrm{P}=0.12)$ in the study population during the fasting of Ramadan (5). Khafaji (7) studied 56 patients with different stable cardiac illnesses and reported that the heart condition was not deteriorated in none of the patients: $71.4 \%$ had no change in their symptoms whereas $28.6 \%$ felt better during Ramadan fast.

Pekdemir et al. (8) observed that during Ramadan, the clinical features of admitted patients and the number of admissions for specific complaints to the Emergency Department did not change significantly as opposed to the 30-day period immediately after Ramadan. More recently, Temizhan et al. (2) compared the incidence of acute coronary syndrome (ACS) in Ramadan with one month before and one month after Ramadan and found that ACS was not more frequent during Ramadan. Furthermore, in patients with CVD, there might be an improvement in ten-year CAD risk score (9).

In contrast, Gumaa et al. (4) reported an increase in complaint of angina pectoris during Ramadan in the 1970s. It has also been reported that during festivals and holidays such as Christmas, cardiac mortality tends to show a significant peak (10). Nonetheless, not only is Ramadan a different holy period from other religious holidays but also it differs from other fasting experiments. During Ramadan, Muslims do not eat and drink for long periods and abstain from smoking, sexual intercourse, and alcohol. Indeed, there is such a pronounced sense of religiousness and spirituality amongst Muslims during this holiest month of the Islamic calendar year that there might directly affect their mental $(11,12)$ and physical health (13). Religious observance might also promote healthy behaviors (14). Hunger has been associated with a decrease in the sympathetic tone and reduced venous return (15), which might lead to a fall in blood pressure $(5,9,15)$, heart rate, and cardiac output (15). A reduction in the heart rate (16-18) and an increase in systolic blood pressure $(17,19)$ have been reported during Ramadan fasting of healthy individuals. This reduced heart rate during Ramadan fasting might be protective in patients with CAD. There might also be an improvement in lipids profile during Ramadan fasting $(9,20)$. A meta-analysis showed that levels of low-density lipoprotein (LDL) and blood glucose (FBS) were decreased and high-density lipoprotein (HDL) increased in both sexes during Ramadan fast in comparison with the levels prior to Ramadan (20). Body mass index (BMI) (5, 9), weight (9, 21), and waist circumference might decrease during Ramadan fast (9); however, a meta-analysis showed that reduction of weight during Ramadan might be relatively small and after Ramadan, most of these weight changes would be reversed and gradually returned to the status before Ramadan (22). It has also been demonstrated that bleeding and coagulation time might increase and platelet responses to the different aggregation agents might decrease by the end of Ramadan (23). These changes might have protective effects against ACS in patients who fast during Ramadan. More studies are required to determine mechanisms for the possible effect of fasting during Ramadan on mental and physical health.

Most of our study population (78.4\%) were treated medically, and $44.8 \%$ of the medically treated patients as well as the patients with a prior CABG or PCI and a history of chest pain who were candidate for medical therapy observed Ramadan fast (Table 1). Lower rates of participation were seen amongst the patients with a history of CABG; around $28 \%$ of the patients with a history of CABG fasted, while $72 \%$ did not. Participation in fasting was more frequent, albeit not statistically significant, in the patients with a history of PCI (54.8\%). The lower participation rates of the patients with a history of CABG could be due to their belief that fasting could have deleterious effects on health.

In the present study, $40.7 \%$ of the patients with hypertension (HTN) and 33.3\% of those with DM observed Ramadan fast (Table 1), and patients with HTN or DM in fasting and non-fasting groups showed no difference in chest pain and a combined endpoint of chest pain and dyspnea (Table 3). Fasting might improve pulse pressure and lipids profile in patients with HTN (24). Perk et al. (25) found that blood pressure control in patients on medications for HTN was not affected by fasting in Ramadan. Sahin et al. studied 122 patients with type 2 DM who fasted during Ramadan and showed that Ramadan fast did not interfere with control of blood glucose in these patients (26) and according to the Benaji et al. (21), patients with DM, who are alert, correctly treated, and monitored closely, can fast without worsening their medical condition. Although our patients with HTN or DM (controlled with oral agents) were able to fast safely during Ramadan, we would suggest more investigations to determine whether Ramadan fast is safe in these subgroups of patients.

\subsection{Study Limitations}

Because we were not confident about the safety of fasting in patients with CAD, ethically we could not randomize the patients to fast. Our study group voluntarily participated in our fasting program and now that we have 
Mousavi Met al.

Table 3. Symptoms of Chest Pain and Combined Chest Pain or Dyspnea in Ninety-One Patients With Hypertension and Thirty-six Patients With Diabetes Mellitus ${ }^{\text {a }}$

\begin{tabular}{|c|c|c|c|c|c|}
\hline Symptom & Fasting Group & Non-Fasting Group & PValue & OR & 95\% CI for OR \\
\hline Chest Pain in Patients With HTN & $2 / 37(5.4 \%)$ & $5 / 54(9.3 \%)$ & 0.696 & 0.560 & 0.103-3.054 \\
\hline $\begin{array}{l}\text { Chest Pain or Dyspnea in Patients } \\
\text { With HTN }\end{array}$ & $2 / 37(5.4 \%)$ & $8 / 54(14.8 \%)$ & 0.192 & 0.329 & $0.066-1.645$ \\
\hline Chest Pain in Patients With DM & $1 / 12(8.3 \%)$ & $1 / 24(4.2 \%)$ & $>0.999$ & 2.091 & $0.119-36.635$ \\
\hline $\begin{array}{l}\text { Chest Pain or Dyspnea in Patients } \\
\text { With DM }\end{array}$ & $1 / 12(8.3 \%)$ & $2 / 24(8.3 \%)$ & $>0.999$ & 1 & $0.081-12.270$ \\
\hline
\end{tabular}

a Abbreviations: OR, odds ratio; CI, confidence interval; HTN, hypertension; and DM, diabetes mellitus.

the results of the present study, a better designed randomized trial could further clarify the safety of Ramadan fast in patients with CAD.

The majority of our patients (78.4\%) were dependent on medical therapy. Our plan to change the pattern of medical treatment with a twice daily schedule might have an effect on symptoms and participation of the patients. Randomization might eliminate this effect. However, most of the patients were able to participate in Ramadan fast without any significant consequences. It seems that most of the drugs can be changed to twice a day, except on rare occasions. Even if the drug itself could not be changed to twice a day, usually there are similar effective drugs with a longer half-life. Physicians should balance the possible benefit of such changes against possible harms.

We excluded patients with systolic left ventricular dysfunction $(\mathrm{EF}<50 \%)$, renal failure, gout, and $\mathrm{DM}$ requiring insulin for treatment or uncontrolled DM. Patients with DM who are treated with insulin might have problems regarding blood glucose control when they fast during Ramadan. Authors also believe that prerenal azotemia and renal failure are the potential harms of Ramadan fast in patients with heart failure and renal failure because these patients must not drink from sunrise to sunset if they fast during Ramadan. Although one study noted that the number of hospitalization for congestive heart failure was not significantly different in Ramadan from those of preceding and following months (27), safety of fasting during Ramadan in patients with heart failure requires more studies and at the present time, authors discourage such patients from Ramadan fast.

In light of our results, we suggest that in patients with CAD and a normal EF, Ramadan fast is not as harmful as it might seem and most of these patients can fast during Ramadan. We would think that it would not be necessary to prevent patients with CAD from Ramadan fast if they are not complicated with significant heart failure, renal failure, and gout and if they do not use insulin for DM control.

\section{Acknowledgements}

We appreciate the valuable assistance of the Mr Pedram Amouzadeh in editing English language of the manuscript.

\section{Authors' Contributions}

Study concept and design: Mehdi Mousavi. Acquisition of data, drafting the manuscript, and critical revision of the manuscript for important intellectual content: Mehdi Mousavi, SadafSadat Mirkarimi, Gita Rahmani, Ehsan Hosseinzadeh, and Navid Salahi. Analysis and interpretation of data: Mehdi Mousavi, SadafSadat Mirkarimi, Ehsan Hosseinzadeh, and Navid Salahi. Statistical analysis, administrative, technical, and material support, and study supervision: Mehdi Mousavi.

\section{References}

1. Sakr AH. Fasting in Islam.J Am Diet Assoc. 1975;67(1):17-21.

2. Temizhan A, Donderici O, Ouz D, Demirbas B. Is there any effect of Ramadan fasting on acute coronary heart disease events? Int J Cardiol.1999;70(2):149-53.

3. Al Suwaidi J, Zubaid M, Al-Mahmeed WA, Al-Rashdan I, Amin $\mathrm{H}$ Bener A, et al. Impact of fasting in Ramadan in patients with cardiac disease. Saudi Med J. 2005;26(10):1579-83.

4. Gumaa KA, Mustafa KY, Mahmoud NA, Gader AM. The effects of fasting in Ramadan. 1. Serum uric acid and lipid concentrations. Br J Nutr. 1978;40(3):573-81.

5. Salim I, Al Suwaidi J, Ghadban W, Alkilani H, Salam AM. Impact of religious Ramadan fasting on cardiovascular disease: a systematic review of the literature. Curr Med Res Opin. 2013;29(4):343-54.

6. Chamsi-Pasha $\mathrm{H}$, Ahmed WH. The effect of fasting in Ramadan on patients with heart disease. Saudi Med J. 2004;25(1):47-51.

7. Khafaji HA, Bener A, Osman M, Al Merri A, Al Suwaidi J. The impact of diurnal fasting during Ramadan on the lipid profile, hs$\mathrm{CRP}$, and serum leptin in stable cardiac patients. Vasc Health Risk Manag. 2012;8:7-14.

8. Pekdemir M, Ersel M, Yilmaz S, Uygun M. No significant alteration in admissions to emergency departments during Ramadan. JEmerg Med. 2010;38(2):253-6.

9. Nematy M, Alinezhad-Namaghi M, Rashed MM, Mozhdehifard M, Sajjadi SS, Akhlaghi S, et al. Effects of Ramadan fasting on cardiovascular risk factors: a prospective observational study. Nutr J. 2012;11:69.

10. Phillips DP, Jarvinen JR, Abramson IS, Phillips RR. Cardiac mortality is higher around Christmas and New Year's than at any other time: the holidays as a risk factor for death. Circulation. 2004;110(25):3781-8.

11. Flannelly KJ, Ellison CG, Strock AL. Methodologic issues in research on religion and health. South Med J. 2004;97(12):1231-41.

12. Ellison CG, Levin JS. The religion-health connection: evidence, theory, and future directions. Health Educ Behav.1998;25(6):700-20.

13. Hill PC, Pargament KI. Advances in the conceptualization and measurement of religion and spirituality. Implications for physical and mental health research. Am Psychol. 2003;58(1):64-74.

14. Koenig HG, George LK, Hays JC, Larson DB, Cohen HJ, Blazer DG. The relationship between religious activities and blood pressure in older adults. Int J Psychiatry Med.1998;28(2):189-213.

15. Stokholm KH, Breum L, Astrup A. Cardiac contractility, central 
haemodynamics and blood pressure regulation during semistarvation. Clin Physiol. 1991;11(6):513-23.

16. Husain R, Duncan MT, Cheah SH, Ch'ng SL. Effects of fasting in Ramadan on tropical Asiatic Moslems. BrJ Nutr.1987;58(1):41-8.

17. Moradmand S, Moosavi M, Nematipour E, Eslami M, Gharouni $\mathrm{M}$, Balali R, et al. Increases of QT dispersion, corrected QT dispersion, and QT interval in young healthy individuals during Ramadan fasting. . Acta Medica Iranica. 2003;41(2):94-9.

18. Ramadan J. Does fasting during Ramadan alter body composition, blood constituents and physical performance? Med Princ Pract. 2002;11 Suppl 2:41-6.

19. Husain R, Cheah SH, Duncan MT. Cardiovascular reactivity in Malay Moslems during Ramadan. Singapore Med J.1996;37(4):398-401.

20. Kul S, Savas E, Ozturk ZA, Karadag G. Does Ramadan fasting alter body weight and blood lipids and fasting blood glucose in a healthy population? A meta-analysis. J Relig Health. 2014;53(3):929-42.

21. Benaji B, Mounib N, Roky R, Aadil N, Houti IE, Moussamih S, et al. Diabetes and Ramadan: review of the literature. Diabetes Res Clin Pract. 2006;73(2):117-25.
22. Sadeghirad B, Motaghipisheh S, Kolahdooz F, Zahedi MJ, Haghdoost AA. Islamic fasting and weight loss: a systematic review and meta-analysis. Public Health Nutr. 2014;17(2):396-406.

23. Aybak M, Turkoglu A, Sermet A, Denli O. Effect of Ramadan fasting on platelet aggregation in healthy male subjects. Eur J Appl Physiol Occup Physiol.1996;73(6):552-6.

24. Al-Shafei AI. Ramadan fasting ameliorates arterial pulse pressure and lipid profile, and alleviates oxidative stress in hypertensive patients. Blood Press. 2014;23(3):160-7.

25. Perk G, Ghanem J, Aamar S, Ben-Ishay D, Bursztyn M. The effect of the fast of Ramadan on ambulatory blood pressure in treated hypertensives. J Hum Hypertens. 2001;15(10):723-5.

26. Sahin SB, Ayaz T, Ozyurt N, Ilkkilic K, Kirvar A, Sezgin H. The impact of fasting during Ramadan on the glycemic control of patients with type 2 diabetes mellitus. Exp Clin Endocrinol Diabetes. 2013;121(9):531-4

27. Al Suwaidi J, Bener A, Hajar HA, Numan MT. Does hospitalization for congestive heart failure occur more frequently in Ramadan: a population-based study (1991-2001). Int J Cardiol. 2004;96(2):217-21. 